\title{
BLOCKS AND NORMAL SUBGROUPS OF FINITE GROUPS
}

\author{
W. F. REYNOLDS
}

To Richard Brauer on his Sixtieth Birthday

\section{Introduction}

Let $H$ be a normal subgroup of a finite group $G$, and let $\zeta$ be an (absolutely) irreducible character of $H$. In [7], Clifford studied the irreducible characters $\chi$ of $G$ whose restrictions to $H$ contain $\zeta$ as a constituent. First he reduced this question to the same question in the so-called inertial subgroup $S$ of $\zeta$ in $G$, and secondly he described the situation in $S$ in terms of certain projective characters of $S / H$. In section 8 of [10], Mackey generalized these results to the situation where all the characters concerned are projective.

Fong proved analogues of both of Clifford's results, in which the characters of $G$ are taken not individually, but in $p$-blocks for a prime $p$. In both of these theorems (Theorems (2 B) and (2 D) of [8]), it is assumed that the order of $H$ is not divisible by $p$.

In section 1, we generalize Fong's first result for arbitrary blocks $B$ and normal subgroups $H$, by defining an inertial subgroup $S$ in $G$ for each block $\widetilde{B}$ of $H$. We show in Theorem 1 that if the restriction to $H$ of any character of $B$ contains a character of $\widetilde{B}$, then the structure of $B$ is identical with that of a corresponding block $B^{\prime}$ of $S$, and the characters of $B$ are induced by those of $B^{\prime}$. In effect, this reduces the whole problem to the case where all the constituents of the restrictions of the characters of $B$ lie in a single block of $H$.

In sections 2 to 4 , we study the second question. The group $S / H$ is no longer appropriate when $p$ divides the order of $H$, since reduction to $S / H$ alters the defect group of the block and thereby destroys the block structure; and we must produce a specially constructed group which will have simpler

Received January 17, 1962.

A portion of this work was done at the Summer Institute for Finite Groups, California Institute of Technology, August, 1960. 
structure than $S$, while retaining the structure of our block. We have succeeded in doing this in the case where the defect group $D$ of $B$ is normal in $G$ and $H$ is its centralizer. (The study of such blocks was begun by Brauer in [3], section 9.) The group $M$ which we construct has a normal $p$-Sylow group, isomorphic to $D$ (see Theorem 6).

In section 5 , we apply this reduction to settle, in the case of a normal defect group, some questions which were raised by Brauer in [2]: Theorem 9 gives a connection between the degrees of the characters of $B$ and the structure of $D$, and Theorem 11 gives a bound on the Cartan invariants of $B$.

Theorem 9 was independently proved by M. Suzuki; I wish to thank him for communicating his proof to me.

Notation. $G$ will always be a group of finite order $g$, and $p$ will be a fixed prime number. We denote by $\nu$ the exponential valuation of the rational field determined by $p$, normalized so that $\nu(p)=1 . \quad \Omega$ is a finite algebraic number field containing the $g$-th roots of unity; $\Omega^{*}$ is the residue class field with respect to a prime divisor $p$ of $p$ in $\Omega$. The (distinct nonequivalent) absolutely irreducible representations of $G$, ordinary and modular, can be written in $\Omega$ and $\Omega^{*}$ respectively; we shall suppose them so written, in definite matricial forms. We shall often suppress the adjective "irreducible".

Each $p$-block $B$ of $G$ is considered here as a set of ordinary irreducible characters, although we shall also make use of the irreducible modular characters of $B$. If $\chi_{j} \in B$, the height of $\chi_{j}$ is the non-negative integer $\nu\left(\chi_{j}(1)\right)-$ $\nu(G: D)$, where the p-group $D$ is a defect group of $B$ (see [3]). We shall pass freely between a representation and its character: for example, we shall sometimes consider $B$ as a set of representations, and speak of the heights of these representations.

\section{Blocks and Induced Characters}

Let $H$ be any normal subgroup of $G$. Then each element $x$ of $G$ permutes the elements $y$ of $H$ by

$$
y \rightarrow y^{x}=x^{-1} y x,
$$

and permutes the irreducible characters $\zeta$ of $H$ by $\zeta \rightarrow \zeta^{x}$, where

$$
\zeta^{x}(y)=\zeta\left(x y x^{-1}\right) .
$$


Characters $\zeta$ and $\zeta^{x}$ so related are called $G$-associates. For each $\zeta,\{x \in G$ : $\left.\zeta^{x}=\zeta\right\}$ is a subgroup of $G$, containing $H$, called the inertial group of $\zeta$ in $G$.

The permutations $\zeta \rightarrow \zeta^{x}$ induce a representation of $G$ by permutations $B \rightarrow B^{x}$ of the $p$-blocks of $H$, by section 1 of [8]. Denote the systems of transitivity of this permutation representation by $\widetilde{\mathscr{T}}_{i}$, anả let $\widetilde{T}_{i}$ be the union of those blocks which are elements of $\widetilde{\mathscr{T}}_{i}$; thus $\widetilde{T}_{i}$ is a set of irreducible characters of $H$.

For each $i$, let $T_{i}$ consist of those irreducible characters $\%_{j}$ of $G$ such that some irreducible constituent of the restriction $\psi_{j} \mid H$ of $\psi_{j}$ to $H$ is in $\widetilde{T}_{i}$. By Lemma (1 B) of [8], the sets $T_{i}$ are disjoint: that is, all the constituents of $\%_{j} \mid H$ are in the same $\widetilde{T}_{i}$; and each $T_{i}$ is a union of $p$-blocks of $G$. We denote the set of those blocks whose union is $T_{i}$ by $\mathscr{T}_{i}$; the same lemma shows that this agrees with Fong's definition of $\mathscr{T}_{i}$.

Now restrict attention to a fixed $\widetilde{T}_{i}=\widetilde{T}$ (dropping subscripts $i$ ), and to a fixed $\widetilde{B} \subseteq \widetilde{T}$. We call the group

$$
S=\left\{x \in G: \widetilde{B}^{x}=\widetilde{B}\right\}
$$

the inertial group of $\widetilde{B}$ in $G$; its index $(G: S)$ is the number of blocks in $\tilde{\mathscr{T}}$.

Take a maximal set $\left\{\zeta_{m}\right\}$ of characters of $\widehat{B}$ such that no two are $G$-associates. By Theorem 1 of [7] we can write $T$ as a disjoint union $\cup F_{m}$, where $F_{m}$ consists of all irreducible characters $\%_{j}$ of $G$ such that $\%_{j} \mid H$ contains $\zeta_{m}$. Let $S_{m}$ be the inertial group of $\zeta_{m}$ in $G$. In section 2 of [7], Clifford sets up a 1-1 correspondence between the characters $\psi_{j}$ of $F_{m}$ and all the irreducible characters $\xi_{j}$ of $S_{m}$ such that $\xi_{j} \mid H$ contains $\zeta_{m}$, in which $\psi_{j}=\xi_{j}^{f}$. Here $\xi_{j}^{f}$ denotes the character of $G$ induced by the character $\xi_{j}$ of the subgroup $S_{m}$ of $G$.

For any $x \in G$ such that $\zeta_{m}^{x} \in \widetilde{B}, \widetilde{B}^{x}=\widetilde{B}$. This implies that $S_{m} \subseteq S$, and that $\left\{\zeta_{m}\right\}$ is also a maximal set of non-S-associate characters of $\widetilde{B}$. If we define $T^{\prime}$ to consist of the irreducible characters $\%_{j}^{\prime}$ of $S$ such that $\chi_{j}^{\prime} \mid H$ contains some character of $\widetilde{B}$, then we have $T^{\prime}=\cup F_{m}^{\prime}$ analogously to the above, and there is a $1-1$ correspondence between the $\chi_{j}^{\prime} \in F_{m}^{\prime}$ and the same $\xi_{j}$ as before, in which $\chi_{j}^{\prime}=\xi_{j}^{s}$.

We can combine these 1-1 correspondences to obtain a 1-1 correspondence $\chi_{j} \leftrightarrow \chi_{j}^{\prime}$ between $F_{m}$ and $F_{m}^{\prime}$, in which $\left(\chi_{j}^{\prime}\right)^{G}=\left(\xi_{j}^{s}\right)^{G}=\xi_{j}^{G}=\chi_{j}$. We have, in fact, 
established a $1-1$ correspondence between $T$ and $T^{\prime}$ in which $\gamma_{i}=\left(\gamma_{j}^{\prime}\right)^{G}$. Let $\mathscr{T}^{\prime}$ be the set of those blocks which make up $T^{\prime}$.

The modular characters of the blocks of $\mathscr{T}$ may be called the modular characters of $T$; they are the modular characters $\phi_{k}$ such that $\phi_{k} \mid H$ contains some modular character of $\widetilde{B}$. Since Clifford's results hold in the modular case, our argument adapts to this case to give a 1-1 correspondence $\phi_{k} \leftrightarrow \phi_{k}^{\prime}$ between the modular characters of $T$ and those of $T^{\prime}$, in which $\phi_{k}=\left(\phi_{k}^{\prime}\right)^{G}$.

The part $\left(d_{j k}^{\prime}\right)$ of the decomposition matrix (see [3] and [5]) of $S$ belonging to the blocks in $\mathscr{T}^{\prime}$ is expressed by the equations

$$
\chi_{j}^{\prime}=\sum_{k} d_{j k}^{\prime} \phi_{k}^{\prime}
$$

where the $\chi_{j}^{\prime}$ and $\phi_{k}^{\prime}$ are the ordinary and modular characters of $T^{\prime}$, and where the relation holds for $p$-regular elements. Inducing to $G$ yields

$$
\varkappa_{j}=\left(\varkappa_{j}^{\prime}\right)^{G}=\left(\sum_{k} d_{j k}^{\prime} \phi_{k}^{\prime}\right)^{G}=\sum_{k} d_{j k}^{\prime}\left(\phi_{k}^{\prime}\right)^{G}=\sum_{k} d_{j k}^{\prime} \phi_{k} .
$$

This means that $\left(d_{j k}^{\prime}\right)$ is also the part $\left(d_{j k}\right)$ of the decomposition matrix of $G$ belonging to the blocks in $\mathscr{T}$. The corresponding statement for the Cartan matrix $\left(c_{k l}\right)$ follows, since $c_{k l}=\sum_{j} d_{j k} d_{j l}$.

Since the Cartan matrices determine the blocks by section 8 of [5], the characters (both ordinary and modular) of each block $B_{\tau} \in \mathscr{T}$ correspond to those of a block $B_{\tau}^{\prime} \in \mathscr{T}^{\prime}$, and conversely. Since $\chi_{j}(1)=(G: S) \%_{j}^{\prime}(1), B_{\tau}$ and $B_{\tau}^{\prime}$ have the same defect. In fact, the argument of Lemma (2 A) of [8] applies almost verbatim to show that each defect group of $B_{\tau}^{\prime}$ is also one for $B_{\tau}$. Finally, the height of $\chi_{j}$ in $B_{\tau}$ is the same as the height of $\chi_{j}^{\prime}$ in $B_{\tau}^{\prime}$.

We summarize in the following theorem, which generalizes Fong's Theorem (2 B).

TheOREM 1. Let $B_{\tau}$ be a block of $G$, and let $H$ be a normal subgroup of $G$. Then there exists a group $S, H \subseteq S \subseteq G$, and a block $B_{\tau}^{\prime}$ of $S$, such that:

(a) the irreducible characters, both ordinary and modular, of $B_{\tau}$ are in 1-1 correspondence with those of $B_{\tau}^{\prime}$, the correspondence being obtained by induction from $S$ to $G$;

(b) $B_{\tau}$ and $B_{\div}^{\prime}$ have the same decomposition matrix, and the same Cartan matrix; 
(c) $B_{\tau}$ and $B_{\tau}^{\prime}$ have a defect group in common;

(d) all the irreducible constituents of the restrictions to $H$ of the characters of $B_{\tau}^{\prime}$ lie in a single block $\widetilde{B}$ of $H$.

We can also show that $B_{\tau}^{\prime}$ and $B_{\tau}$ stand in the relation defined by Brauer in section 2 of [4]. This is included in the following general result, which is independent of the assumptions of this section.

TheOREm 2. Let $B^{\prime}$ be a block of a subgroup $S$ of a group $G$, such that $B^{\prime}$ contains some character $\chi^{\prime \prime}$ such that $\%=\left(\chi^{\prime}\right)^{G}$ is irreducible. Then $\left(B^{\prime}\right)^{G}$ is defined (in the notation of $[4])$, and $\% \in\left(B^{\prime}\right)^{G}$.

Proof. If $\omega^{\prime}$ and $\omega$ are the characters of the class-algebras of $S$ and $G$ over $\Omega$ corresponding to $\chi^{\prime}$ and $\chi$ respectively, it follows from the definitions that

$$
\omega\left(K_{a}\right)=\sum_{K_{\beta}^{\prime} \subseteq K \alpha} \omega^{\prime}\left(K_{\beta}^{\prime}\right),
$$

where $K_{\alpha}$ and $K_{\beta}^{\prime}$ are arbitrary conjugate classes of $G$ and $S$ respectively. Taking this relation modulo $\mathfrak{p}$, we get the result.

\section{Defect Group in Center}

We begin our study of blocks with normal defect group by considering the case where the defect group is in the center of the group. (In this section, the field $\Omega$ in which all elements and representations lie is to contain the $(H: 1)$ roots of unity.)

Assume that $B$ is a $p$-block of a group $H$ such that the defect group $A$ of $B$ is contained in the center of $H$. The normal $p$-subgroup $A$ lies in the kernel of every irreducible modular representation $\widetilde{\mho}_{k}$ of $H$ (by (9 D) of [3]), so that these can be considered as modular representations of $H / A$. They are partitioned into $p$-blocks in the same way for $H$ as for $H / A$, as can be seen by making use of $(2 \mathrm{G})$ of reference [4]. Now $B$ contains some $\widetilde{f}$ such that $\nu(\mathrm{deg}$ $\mathfrak{F})=\nu(H)-\nu(A)$ where $\operatorname{deg} \mathfrak{F}$ is the degree of $\mathfrak{F}$, by $(6 \mathrm{~A})$ of [3]; taken on $H / A, \widetilde{F}$ belongs to a block of defect 0 , by $(2 \mathrm{G})$ of [4]. By the properties of such blocks, this is the only modular representation in $B$; and there is an ordinary representation 3 in $B$ such that $3^{*}=\mathfrak{F}$. Here we write 3 with matrix coefficients which are locally integral at $\mathfrak{p}$, and $3^{*}$ is obtained by reducing 
these coefficients modulo $\mathfrak{p}$. Denote the characters of 3 and $\tilde{F}$, on $H$, by $\zeta$ and $\phi$ respectively.

Since $\zeta$ belongs to a block of defect 0 of $H / A$, it vanishes for all $p$-singular elements of $H / A$. In terms of $H$, this means that

$$
\zeta(x)=0 \quad \text { if } x \in H, x_{p} \notin A,
$$

where we write the factorization of $x$ into a $p$-element $x_{p}$ and a $p$-regular element $x_{r}$ as $x=x_{p} x_{r}=x_{r} x_{p}$.

For the character $\zeta_{j}$ of any representation $3_{i}$ in $B$ (taking $\zeta=\zeta_{1}$ ), we have

$$
\zeta_{j}(x)=d_{j} \phi(x)=d_{j} \zeta(x) \quad \text { if } x \text { is } p \text {-regular, }
$$

since $\phi$ is the only modular character in $B$; the positive integers $d_{j}$ are the decomposition numbers of $B$. For $x$ such that $x_{p} \in A, 3_{j}(x)=3_{j}\left(x_{p}\right) 3_{j}\left(x_{r}\right)$ $=\omega_{j}\left(x_{p}\right) 3_{j}\left(x_{r}\right)$, where $\omega_{j}$ is a character of the abelian group $A$, depending on $3 j$. By (5),

$$
\zeta_{j}(x)=\omega_{j}\left(x_{p}\right) \zeta_{j}\left(x_{r}\right)=d_{j} \omega_{j}\left(x_{p}\right) \zeta\left(x_{r}\right)=d_{j} \omega_{j}\left(x_{p}\right) \zeta(x)
$$

whenever $x_{p} \in A$.

Now apply the orthogonality relations on $H$, setting $(H: 1)=h$ :

$$
1=h^{-1} \sum_{x \in H}\left|\zeta_{j}(x)\right|^{2}=h^{-1} \sum_{x: x_{p} \in A}\left|d_{j} \omega_{j}\left(x_{p}\right) \zeta(x)\right|^{2}+h^{-1} \sum_{x: x_{p} \notin A}\left|\zeta_{j}(x)\right|^{2} .
$$

If we denote the two terms on the right by $s_{j 1}$ and $s_{j 2}$, (4) implies that $0<s_{j 1}$ $=d_{j}^{2} h^{-i} \sum_{x}|\zeta(x)|^{2}=d_{j}^{2}=1-s_{j 2}$, hence $d_{j}=1, s_{j_{2}}=0$. That is,

$$
\zeta_{j}(x)= \begin{cases}\omega_{j}\left(x_{p}\right) \zeta(x) & \text { if } x_{p} \in A, \\ 0 & \text { if } x_{p} \notin A .\end{cases}
$$

If we denote all the characters of $A$ by $\omega_{j}, 1 \leq j \leq(A: 1),(6)$ shows that each $\omega_{j}$ is associated with at most one character of $B$. Let $J$ consist of those indices $j$ such that the function $\zeta_{j}$ on $H$ defined by $(6)$ is actually a character of $B$. Then the principal indecomposable character $\Phi$ corresponding to $\phi$ is given by $\mathscr{D}=\sum_{j \in J} d_{j} \zeta_{j}=\sum_{j \in J} \zeta_{j}$, whence $\mathscr{D} \mid A=\zeta(1) \sum_{j \in J} \omega_{j}$ (see section 3 of [3]). But $\Phi$ vanishes for all $p$-singular elements, so that $\Phi \mid A$ is a multiple of the regular representation of $A$. Comparing these expressions, we see that all indices from 1 to $(A: 1)$ are in $J$. This completes the proof of the following theorem. 
Theorem 3. Let $B$ be a block of a finite group $H$, whose defect group $A$ is contained in the center of $H$. Then $B$ contains just one modular character and $(A: 1)$ ordinary characters, all of the same degree. Each of the ordinary characters is associated with a character of $A$ by the equations (6).

Since $\zeta_{j}(x)=\phi(x)$ for $p$-regular $x$, we can write $3_{j}$ with locally integral coefficients in such a way that $3_{j}^{*}=3^{*}=\widetilde{F}$.

We shall need the following form of Theorem 3 for projective representations. (See [1], [11], [12] for background on projective representations.)

THEOREM 4. Let $A_{0}$ be a p-subgroup of the center of a finite group $X$, and let $\varepsilon$ be a factor set of $X / A_{0}$ whose values are roots of unity of orders prime to $p$. Let $\mathfrak{Y}$ be an irreducible projective representation of $X / A_{0}$ with factor set $\varepsilon$, written with locally integral coefficients, such that $\nu(\operatorname{deg} Y)=\nu\left(X: A_{0}\right)$. Then for each linear character $\omega_{0 j}$ of $A_{0}$, there exists a projective representation $\mathfrak{Y}_{j}$ of $X$ for $\varepsilon$, with locally integral coefficients, such that $\mathfrak{Y}^{*}=\mathfrak{Y}_{j}^{*}$ and $\mathfrak{Y}_{j} \mid A_{0}$ is a multiple of $\omega_{0 j}$.

Observe that we regard $\mathfrak{Y}$ as a representation of $X$, and $\varepsilon$ as a factor set of $X$ by inflation, without special mention. For the trivial factor set this theorem becomes a restatement of Theorem 3 in a form which avoids mentioning blocks. We can assume that $\varepsilon_{x, y}=1$ whenever either $x$ or $y$ lies in $A_{0}$.

Proof. We reduce this to Theorem 3 by the classical method of Schur (see [1], and also page 274. of [8]). Let $E$ be the character group of the multiplicative cyclic group generated by the factor set $\varepsilon ; E$ is cyclic of order prime to $p$. For any elements $x, y$ of $X$, let $m_{x, y} \in E$ be the character such that $\varepsilon^{i} \rightarrow\left(\varepsilon_{x}, y\right)^{i}$; then $m=\left\{m_{x, y}\right\}$ is a factor set of $X$ with values in $E$. Using extension theory (see [13]), let $H$ be the extension of $E$ by $X$ with this factor set and trivial action; the elements of $H$ may be written $(e, x)$, where $e \in E$, $x \in X$. The elements $(1, a), a \in A_{0}$, form a subgroup $A$ of the center of $H$ ( since $\varepsilon$ is trivial on $A_{0}$ ), isomorphic to $A_{0}$. $A$ character $\omega_{j}$ of $A$ is defined by $\omega_{j}(1, \boldsymbol{a})=\omega_{0},(\boldsymbol{a})$; and the equation $3(\boldsymbol{e}, \boldsymbol{x})=\boldsymbol{e}(\varepsilon) \mathfrak{Y}(x)$ defines an ordinary representation 3 of $H$, which can be regarded as belonging to a block of $H / A$ of defect 0 . Now Theorem 3 provides us with a representation $3 j$ of $H$ such that $3_{j}^{*}=3^{*}$ and $3_{j} \mid A$ is a multiple of $\omega_{j}$. The required $\mathfrak{Y}_{j}$ is then defined by $3_{j}(e, x)=e(\varepsilon) \mathfrak{Y}_{j}(x)$, 


\section{Normal Defect Group: Analysis}

In this section, we shall analyze the representations in a block whose defect group is normal. The information so obtained will be the basis for the constructions of the following section.

Let $D$ be a normal $p$-subgroup of $G$. Denote the centralizer of $D$ by $H$, and the center of $D$ by $A$; then $A=D \cap H$, and $H$ and $A$ are normal in $G$. By (11 B) of [3], there is a 1-1 correspondence between the blocks $B$ of $G$ with defect group $D$ and the families of $G$-associates of these characters $\zeta$ in blocks of defect 0 of $H / A$ such that $p+(S: D H)$, where $S / A$ is the inertial group of $\zeta$ in $G / A$. (This restatement uses the isomorphism of $D H / D$ with $H / A$.) In addition, $D$ must be the maximal normal $p$-subgroup of $G$ in order for any blocks with normal defect group $D$ to exist, since this subgroup is contained in the defect group of every block of $G$.

Henceforth, we assume that we have a block $B$ with normal defect group $D$; we study it along with a corresponding $\zeta$ and $S$, as just defined. We can regard $\zeta$ as a character of $H$. Since $A$ is contained in the center of $H,(2 \mathrm{G})$ of [4] tells us that $\zeta$ belongs to a block $\widetilde{B}$ of $H$ with defect group $A$, and Theorem 3 shows that the characters of $\widetilde{B}$ have the form $\zeta_{l}$, one for each character $\omega_{l}$ of $A$. Here $\zeta=\zeta_{1}$ corresponds to the 1 -character of $A$.

Choose a maximal set $\left\{\omega_{m}\right\}$ of non-G-associate characters of $A$. Then by (6), $\left\{\zeta_{m}\right\}$ is a maximal set of non-G-associate characters of $\widetilde{B}$. Let $S_{m}$ be the inertial group of $\zeta_{m}$ in $G$, as in section 1 . Then $S_{m}$ is the intersection of $S=S_{1}$ with the inertial group of $\omega_{m}$ in $G$. Since $\zeta$ yields the only modular character of $\widetilde{B}, S$ is the inertial group of $\widetilde{B}$ in $G$, defined in (3).

In the terminology of section $1, B \in \mathscr{T}$ where $\widetilde{B} \in \widetilde{\mathscr{T}}$; this follows from equation (11.11) of [3], and it enables us to study the corresponding block $B^{\prime} \in \mathscr{T}^{\prime}$ instead of $B . \quad B$ is actually the only block in $\mathscr{T}$, by the following argument. For any $B_{\tau} \in \mathscr{T}$, let $\chi_{j}^{\prime}$ be an arbitrary character of $B_{\tau}^{\prime}$. Since $\chi_{j}^{\prime} \mid H$ contains some $\zeta_{m}, \zeta(1)=\zeta_{m}(1) \mid \chi_{j}^{\prime}(1)$ by [7], whence $\nu\left(\chi_{j}^{\prime}(1)\right) \geq \nu(\zeta(1))$ $=\nu(D H: D)=\nu(S: D)$, so that the defect of $B_{\tau}^{\prime}$ does not exceed ${ }_{\nu}(D: 1)$. But since $D$ is contained in all defect groups for blocks of $S, D$ is the defect group for $B_{\tau}^{\prime}$ and hence for $B_{\tau}$ also. But we have previously accounted for all blocks with defect group $D$, and none of them except $B$ is in $\mathscr{T}$; therefore

$$
\mathscr{T}=\{B\} .
$$


Accordingly, $\mathscr{T}^{\prime}=\left\{B^{\prime}\right\}, B=\cup F_{m}$, and $B^{\prime}=\cup F_{m}^{\prime}$.

Since $S / H$ has normal Sylow subgroup $D H / H$, there exists a subgroup $K$ of $S$ with $S=(D H) K, H=D H \cap K$, by a theorem of Schur (see [13], p. 132). Then $S=D K$, and $D \cap K=D \cap D H \cap K=D \cap H=A$.

Let us regard $S$ as an extension of the abelian group $A$ by $\bar{S}=S / A$. For each $\jmath \in \bar{S}$, choose a representative $s_{o} \in S$. Since $\bar{S}=\bar{D} \bar{K}, 1=\bar{D} \cap \bar{K}$ where $\bar{D}=D / A, \bar{K}=K / A$, we can suppose that

$$
s_{\delta \kappa}=s_{\delta} s_{\kappa}
$$

when $\delta \in \bar{D}, \kappa \in \bar{K}$, and that $s_{1}=1$. For any $a \in A$ and $\sigma \in \bar{S}$ set

$$
a^{\sigma}=a^{s \sigma} \text {. }
$$

Then for any $\sigma, \tau \in \bar{S}$,

$$
s_{\sigma} s_{\tau}=r_{\sigma, \tau} s_{\sigma \tau},
$$

where $r=\left\{\boldsymbol{r}_{\sigma, \tau}\right\}$ is a factor set of $\bar{S}$ with values in $A$ : that is,

$$
\boldsymbol{r}_{\sigma, \tau} \boldsymbol{r}_{\sigma \tau, v}=\boldsymbol{r}_{\tau, v}^{\sigma^{-1}} \boldsymbol{r}_{\sigma, \tau v} \quad \text { for } \sigma, \tau, v \in \bar{S} \text {. }
$$

For $\sigma, \tau \in \bar{S}_{m}=S_{m} / A$, set $\left(\rho_{m}\right)_{o, \tau}=\omega_{m}\left(r_{\sigma, \tau}\right)$. Since $S_{m}$ is contained in the inertial group of $\omega_{m}$, (11) yields $\left(\rho_{m}\right)_{\sigma, \tau}\left(\rho_{m}\right)_{\sigma \tau, v}=\left(\rho_{m}\right)_{\tau, v}\left(\rho_{m}\right)_{\sigma, \tau v}$ for $\sigma, \tau, v \in \bar{S}_{m}$. That is, $\rho_{m}=\left\{\left(\rho_{m}\right)_{\sigma, \tau}\right\}$ is a factor set of $\bar{S}_{m}$ with trivial action, the values being roots of unity in $\Omega$ whose orders are powers of $p$. For $a \in A, \sigma \in \bar{S}_{m}$, set $\phi_{m}\left(a s_{\sigma}\right)=\omega_{m}(a)$. Then $\psi_{m}\left(a s_{\sigma} b s_{\tau}\right)=\psi_{m}\left(a b^{\sigma^{-1}} \boldsymbol{r}_{\sigma, \tau} \boldsymbol{s}_{\sigma \tau}\right)=\omega_{m}\left(a b^{\sigma^{-1}} \boldsymbol{r}_{\sigma, \tau}\right)=$ $\omega_{m}(a) \omega_{m}(b) \omega_{m}\left(r_{\sigma, \tau}\right)=\left(\rho_{m}\right)_{\sigma, \tau} \psi_{m}\left(a s_{\sigma}\right) \psi_{m}\left(b s_{\tau}\right)$, so that $\psi_{m}$ is a projective representation of $\bar{S}_{m}$ of degree 1, with factor set $\rho_{m}^{-1}$. Here $\rho_{m}$ is regarded as a factor set of $S_{m}$, by inflation; as usual, we do not indicate this inflation explicitly.

From now on, we shall work in terms of representations rather than the corresponding characters. By the remark following Theorem 5, we suppose that $3_{m}^{*}=3^{*}$, where $3_{m}$ is the representation of $H$ corresponding to $\zeta_{m}$.

By section 3 of [7] (cf. [10]), there must exist ${ }^{11}$ a projective representation of $S_{m}$ whose restriction to $H$ is $3 m$, and whose factor set, which we shall call $\varepsilon_{m}^{-1}$, is inflated from $S_{m} / H$. In order to compute $\varepsilon_{m}$ in terms of $\varepsilon=\varepsilon_{1}$ (see (14)), we first restrict attention to $K$. We can assume that the values taken on by $\varepsilon \mid K$ are roots of unity in $\Omega$, of orders prime to $p$ (see [1]).

1) At this point, and later on, we may have to replace $\Omega$ by a finite extension of $\Omega$, since [7] uses an algebraically closed field. But in fact these extensions can be avoided, as the addendum at the end of this paper shows. 
Let $\mathfrak{Y}$ be a projective representation of $K$, whose factor set is the restriction $\varepsilon^{-1} \mid K$ of $\varepsilon^{-1}$ to $K$, such that $\mathfrak{Y} \mid H=3$, and such that $\mathfrak{Y}$ has locally integral coefficients. Let $J_{m} \subseteq A$ be the kernel of $\omega_{m}$, and let $K_{m}=S_{m} \cap K$. Then all commutators $a^{-1} s^{-1} a s, a \in A, s \in K_{m}$, are in $J_{m}$, so that $A / J_{m}$ is contained in the center of $K_{m} / J_{m}$. Since $\nu(\operatorname{deg} \mathfrak{Y})={ }_{\nu}\left(K_{m}: A\right)$, we can apply Theorem 4 to $\mathfrak{Y} \mid K_{m}$, regarded as a projective representation of $X=K_{m} / J_{m}$, to find that $K_{m}$ has a projective representation $\mathfrak{Y}_{m}$ with factor set $\varepsilon^{-1} \mid K_{m}$, such that $\mathfrak{Y}_{m} \mid A$ is a multiple of $\omega_{m}$, and such that $\mathfrak{Y}_{m}^{*}=\mathfrak{Y}^{*} \mid K_{m}$. Then $\mathfrak{Y}_{m} \mid H$ is equivalent to $\mathfrak{Z}_{i}$, and we may suppose that $\mathfrak{Y}_{m} \mid H=3_{m}$.

We extend $\mathfrak{Y}_{m}$ to $S_{m}$ by the following steps. First, write $\mathfrak{Y}_{m}=\left(\psi_{m} \mid K_{m}\right) \overline{\mathfrak{Y}}_{m}$, where $\overline{\mathfrak{Y}}_{m}$ is a projective representation of $K_{m}$ with factor set $\left(\varepsilon^{-1} \mid K_{m}\right)\left(\rho_{m} \mid K_{m}\right)$ which can also be regarded as a projective representation of $\bar{K}_{m}=K_{m} / A$. This can be seen by section 8 of [10], or directly. (For finite groups, the topological assumptions of [10] are vacuously satisfied, and the restriction to unitary representations is unnecessary.) Secondly, since $\bar{S}_{m}=\bar{D} \bar{K}_{m}, 1=\bar{D} \cap \bar{K}_{m}$, we have a natural isomorphism of $\bar{S}_{m} / \bar{D}$ with $\bar{K}_{m}$; use this to carry over $\overline{\mathfrak{Y}}_{m}$ to $\bar{S}_{m} / \bar{D}$. Thirdly, inflate to $\bar{S}_{m}$; this yields an extension of the original $\overline{\mathfrak{Y}}_{m}$, for which we retain the same symbol. Finally, set $\mathfrak{Y}_{m}=\psi_{m} \overline{\mathfrak{Y}}_{m}$ on $S_{m}$. This gives a projective representation $\mathfrak{Y}_{m}$ of $S_{m}$, related to its restriction on $K_{m}$ by $\bigvee_{m}\left(a s_{\delta \kappa}\right)=\mathfrak{Y}_{m}\left(a s_{\kappa}\right)$ where $a \in A, \delta \in \bar{D}, \kappa \in \bar{K}_{m}$. Modularly, $\bigvee_{m}^{*}=\mathfrak{Y}^{*} \mid S_{m}$.

The factor set of $\bigvee_{m}$ is readily seen to be $\left(\varepsilon^{-1} \mid K_{m}\right) \mu_{m}^{-1}$, where $\mu_{m}$ can be defined as a factor set on $\bar{S}_{m}$ by

$$
\left(\mu_{m}\right)_{\delta \kappa, \gamma \lambda}=\left(\rho_{m}\right)_{\delta \kappa, \gamma \lambda}\left(\rho_{m}\right)_{\kappa, \lambda}^{-1}=\omega_{m}\left(\boldsymbol{r}_{\delta \kappa, \gamma \lambda} \boldsymbol{r}_{\kappa, \lambda}^{-1}\right)
$$

for $\delta, \gamma \in \bar{D}, \kappa, \lambda \in \bar{K}_{m}$. Now for any $\delta, \gamma \in \bar{D}, \kappa, \lambda \in \bar{K}$, we have by (8) and (10) $r_{\delta \kappa, \gamma \lambda}=s_{\delta \kappa} s_{\gamma \lambda} s_{\delta r_{1} \kappa \lambda}^{-1}=s_{\delta} s_{\kappa} s_{\gamma} s_{\lambda} s_{\kappa \lambda}^{-1} s_{\delta r_{1}}^{-1}$, where we set $\gamma_{1}=\gamma^{k^{-1}}$. Since $r_{\kappa . \lambda} \in A$ and $s_{\delta r_{1}} \in D$,

$$
\begin{aligned}
q_{\delta \kappa, \Upsilon \lambda} & =r_{\delta \kappa, \Upsilon \lambda} r_{\kappa, \lambda}^{-1}=s_{\delta} s_{\kappa} s_{\curlyvee} s_{\lambda} s_{\kappa \lambda}^{-1} r_{\kappa, \lambda}^{-1} s_{\delta \Upsilon_{1}}^{-1} \\
& =s_{\delta}\left(s_{\kappa} s_{\curlyvee} s_{\kappa}^{-1}\right)\left(s_{\kappa} s_{\lambda} s_{\kappa \curlywedge}^{-1} r_{\kappa, \lambda}^{-1}\right) s_{\delta \gamma_{1}}^{-1}=s_{\delta}\left(s_{\curlyvee}\right)^{-1} s_{\delta \gamma_{1}}^{-1} .
\end{aligned}
$$

Since $D$ commutes elementwise with $H$, the last expression shows that $q_{\delta \kappa}, \because \lambda$ does not change if we multiply $\kappa$ and $\lambda$ by elements of $\bar{H}=H / A$. Therefore $q=\left\{q_{\sigma, \tau}\right\}$, which is a factor set of $\bar{S}$ with values in $A$ under the same action as for $r$ ( $S / H)$, under the action of $\bar{S} / \bar{H}$ on $A$ defined by 


$$
a^{\sigma \bar{H}}=a^{\sigma}
$$

(cf. (9)). Since

$$
\left(\mu_{m}\right)_{\sigma, \tau}=\omega_{m}\left(q_{\sigma, \tau}\right), \quad \sigma, \tau \in \bar{S}_{m},
$$

$\mu_{m}$ is inflated from a factor set of $S_{m} / H$ with values in $\Omega$ and trivial action. Therefore

$$
\varepsilon_{m}=\left(\varepsilon \mid S_{m}\right) \mu_{m}
$$

is inflated from $S_{m} / H$, and $\mathfrak{Y}_{m}$ is an extension of $3_{m}$, with factor set $\varepsilon_{m}^{-1}$.

Theorem 3 of [7] sets up a 1-1 correspondence between the representations $\mathfrak{X}_{j}^{\prime}$ corresponding to all the $\chi_{j}^{\prime} \in F_{m}^{\prime}$ (that is, all representations of $S$ whose restrictions to $H$ contain $3_{m}$ ) and all projective representations $\mathfrak{A}_{j}$ of $S_{m} / H$ with factor set $\varepsilon_{m}$, such that

$$
\mathfrak{X}_{j}^{\prime}=\left(\mathfrak{Y}_{m} \times \mathfrak{A}_{j}\right)^{S} .
$$

Here we treat $\mathfrak{A}_{j}$ as a projective representation of $S_{m}$, so that the tensor product $\mathfrak{Y}_{m} \times \mathfrak{R}_{j}$ is an ordinary representation of $S_{m}$, which is induced to $S$ to yield $\mathfrak{X}_{j}^{\prime}$. We can suppose that $\mathfrak{A}_{j}$ has locally integral coefficients. By Theorem 1 , we also have $\mathfrak{X}_{j}=\left(\mathfrak{Y}_{m} \times \mathfrak{U}_{j}\right)^{G}$, for $\mathfrak{X}_{j} \in F_{m}$.

Now consider the situation in $\Omega^{*}$. $\mathfrak{U}_{j}^{*}$ is a modular projective representation of $S_{m} / H$, whose factor set $\varepsilon_{m}^{*}$ is obtained by reducing $\varepsilon_{m}$ modulo p. By (14), $\varepsilon_{m}^{*}=\varepsilon^{*} \mid S_{m}$, since the values of $\mu_{m}$ are roots of unity of $p$-power orders. By (15),

$$
\left(\mathfrak{X}_{j}^{\prime}\right)^{*}=\left(\mathfrak{Y}_{m}^{*} \times \mathfrak{A}_{j}^{*}\right)^{s}=\left(\left(\mathfrak{Y}^{*} \mid S_{m}\right) \times \mathfrak{A}_{j}^{*}\right)^{s} \sim \mathfrak{Y}^{*} \times\left(\mathfrak{U}_{j}^{*}\right)^{s},
$$

where the meaning of and reason for the last relation are as follows. $\left(\mathfrak{R}_{j}^{*}\right)^{s}$ is the modular projective representation of $S$ with factor set $\varepsilon^{*}$ induced by $\mathfrak{Y}_{j}^{*}$ (see [10], [11], [12] for definition). The symbol $\sim$ indicates that the modular representations of $S$ which it joins have the same irreducible constituents and multiplicities. That this is true is basically a consequence of the Frobenius reciprocity theorem and the orthogonality relations; a proof can be constructed in the following way. Express everything in terms of modular projective characters, with the factor sets $\varepsilon$ and $\varepsilon \mid S_{m}$, whose values are roots of unity in $\Omega$ of orders prime to $p$. Extend each such character by defining its values for $p$-singular elements to be zero, and express it as a rational linear combina- 
tion of irreducible projective characters in $\Omega$ with the same factor set. By linearity, it now suffices to prove the corresponding statement for irreducible projective characters in $\Omega$. But this follows from Theorem 4.6 of [10]. (Cf. [11], and equation (4) of [6].)

The irreducible modular representations $\widetilde{f}_{k}^{\prime}$ which are in $B^{\prime}$ are precisely those such that $\widetilde{F}_{k}^{\prime} \mid H$ contains $3^{*}$. Since the inertial group of the character of $3^{*}$ is $S$ and since Clifford's results still hold in the modular case, we have a 1-1 correspondence between these $\mathfrak{F}_{k}^{\prime}$ and all the irreducible modular projective representations $\mathfrak{B}_{k}$ of $S / H$ with factor set $\varepsilon^{*}$ (or, as we may say, the $\varepsilon^{*}$-representations of $S / H$ ) in which $\mathfrak{F}_{k}^{\prime}=\mathfrak{Y}^{*} \times \mathfrak{B}_{k}$. In $B$, the corresponding equation is $\widetilde{\jmath}_{k}=\left(\mathfrak{Y}^{*} \times \mathfrak{B}_{k}\right)^{G}$.

Since $\mathfrak{A l}_{j}^{*}$ can be regarded as a projective representation of $S_{m} / H$, we can write formally

$$
\left(\mathfrak{A}_{j}^{*}\right)^{S} \sim \sum_{k} d_{j k} \mathfrak{B} k
$$

for some non-negative integers $d_{j k}$. Combining our results, we find that

$$
\left(\mathfrak{X}_{j}^{\prime}\right)^{*} \sim \mathfrak{Y}^{*} \times \sum_{k} d_{j k} \mathfrak{B}_{k} \sim \sum_{k} d_{j k}\left(\mathfrak{Y}^{*} \times \mathfrak{B}_{k}\right)=\sum_{k} d_{j k} \mathfrak{F}_{k}^{\prime} .
$$

Using all values of $m$, this shows that the numbers $d_{j k}$ are precisely the decomposition numbers for $B^{\prime}=\cup F_{m}^{\prime}$, and hence also for $B$, in agreement with the notation of section 1 .

\section{Normal Defect Group: Construction}

In the previous section, we defined a factor set $q$ of $\bar{S} / \bar{H}$ with values in $A$, under the action defined by (12). Let $U$ be the extension of $A$ by $\bar{S} / \bar{H}$ defined by this action and factor set. We may write the elements of $U$ as ordered pairs $u=(a, \sigma \bar{H})$, where $a \in A$ and $\sigma \in \bar{S}$. Denote the subgroup $\{(a$, $\bar{H})\}$ by $A^{0}$; and let $i$ be the natural isomorphism of $\bar{S} / \bar{H}$ onto $U / A^{0}$. The elements $(a, \delta \bar{H}), \delta \in \bar{D}$, form a subgroup $D^{0}$ of $U$ isomorphic to $D$ under the mapping $(a, \delta \bar{H}) \rightarrow a s_{\delta}$, since $q|D=s| D$. Since $i(\bar{D} \bar{H} / \bar{H})=D^{0} / A^{0}$, we have the isomorphisms $U / D^{0} \cong \bar{S} / \bar{D} \bar{H} \cong S / D H$, so that $D^{0}$ is a normal $p$-Sylow subgroup of $U$. A $p$-complement in $U$ is then given by $L^{0}=\{(1, \kappa \bar{H}): \kappa \in \bar{K}\} \cong K / H$ : thus $U=D^{0} L^{0}, 1=D^{0} \cap L^{0}$. The action of $L^{0}$ on $D^{0}$ by conjugation can be computed straightforwardly; it corresponds in the natural way to the action 
of $K / H$ on $D$ in $S$. Hence it follows that $D^{0}$ contains its own centralizer in $U$, namely $A^{0}$. Thus the structure of $U$ is analogous to that of $S$, but is more definite; the analogues of $H$ and $K$ are $A^{0}$ and $K^{0}=A^{0} L^{0}$ respectively.

The factor set $\varepsilon$, regarded as a factor set of $\bar{S} / \bar{H}$, can be carried over by $i$ to a factor set $\varepsilon^{0}$ of $U / A^{0}$, which can then be inflated to $U$. In the same way, we can use $\varepsilon_{m}$ and $\mu_{m}$ to define factor sets $\varepsilon_{m}^{0}$ and $\mu_{m}^{0}$ of $S_{m}^{0}$, where $i\left(\bar{S}_{m} / \bar{H}\right)=S_{m}^{0} / A^{0}$ (cf. (13)). The equation $\psi_{m}^{0}(a, \sigma \bar{H})=\omega_{m}(\boldsymbol{a})$ defines a projective representation of $S_{m}^{0}$ with factor set $\left(\mu_{m}^{0}\right)^{-1}$. Then $\psi_{m}^{0} \mid A^{0}=\omega_{m}^{0}$ corresponds to $\omega_{m}$ under the natural isomorphism of $A$ and $A^{0}$.

We now study the $\varepsilon^{0}$-representations $\mathfrak{X}_{j}^{0}$ of $U$ in analogy with the study of the representations of $B$ in section 3 . Since $\varepsilon^{0} \mid A^{0}$ is trivial, the $\mathfrak{X}_{j}^{0}$ can be distributed among disjoint sets $F_{m}^{0}$ according to which $\omega_{m}^{0}$ is contained in $\mathfrak{X}_{j}^{0}$; note that there is no question of blocks in this definition. The inertial group of $\omega_{m}^{0}$ in $U$ is $S_{m}^{0}$, even with respect to $\varepsilon^{0}$ in the sense of Mackey ([10], Theorem 8.1). Then Theorem 8.4 of [10] gives a 1-1 correspondence between the $\mathfrak{X}_{j}^{0} \in F_{m}^{0}$ and all the representations $\mathfrak{H}_{j}^{0}$ of $S_{m}^{0} / A^{0}$ for $\varepsilon_{m}^{0}=\left(\varepsilon^{0} \mid S_{m}^{0}\right) \mu_{m}^{0}$, in which

$$
\mathfrak{X}_{j}^{0}=\left(\psi_{m}^{0} \mathfrak{A}_{j}^{0}\right)^{U},
$$

the induction being with respect to $\varepsilon^{0}$. We can suppose that $\mathfrak{H}_{j}^{0}$ has been obtained from the $\mathfrak{A}_{j}$ of (15) by means of $i$. Thus we have a 1-1 correspondence $\mathfrak{X}_{j} \leftrightarrow \mathfrak{X}_{j}^{0}$ between $F_{m}$ and $F_{m}^{0}$, hence between the ordinary representations of $B$ and all the $\varepsilon^{0}$-representations of $S$.

The $\left(\varepsilon^{0}\right)^{*}$-representations $\mathfrak{F}_{k}^{0}$ of $U$ all have kernels containing $A^{0}$, as we see by generalizing ( $9 \mathrm{D})$ of [3] to the projective case-cf. the construction of $M$ below. Thus we can suppose that $\mathscr{\gamma}_{k}^{0}$, as an $\left(\varepsilon^{0}\right)^{*}$-representation of $U / A^{0}$, corresponds to $\mathfrak{B}_{k}$ under $i$. This gives a $1-1$ correspondence $\mathfrak{F}_{k} \leftrightarrow \mathfrak{F}_{k}^{0}$.

Applying $i$ to (16) yields $\left(\left(\mathfrak{H}_{j}^{0}\right)^{*}\right)^{U} \sim \sum_{k} d_{j k} \widehat{\mho}_{k}^{0}$. Since the values of $\phi_{m}^{0}$ are all $p$-power roots of unity, $\left(\psi_{m}^{0}\right)^{*}=1^{*}$, so that $\left(\mathfrak{X}_{j}^{0}\right)^{*}=\left(\left(\mathfrak{A}_{j}^{0}\right)^{*}\right)^{U} \sim \sum_{k} d_{j k} \mathfrak{F}_{k}^{0}$, where the $d_{j k}$ are again the decomposition numbers of $B$. We have proved the following theorem.

Theorem 5. Let $B$ be a block of a group $G$, with normal defect group $D$. Then there exists a group $U$ and a factor set $\varepsilon^{0}$ of $U$, whose values are roots of unity of orders prime to $p$, such that:

(a) the ordinary representations of $B$ are in 1-1 correspondence with the 
$\varepsilon^{0}$-representations of $U$, the modular representations of $B$ are in 1-1 correspondence with the $\left(\varepsilon^{0}\right)^{*}$-representations of $U$, and corresponding representations have proportional degrees and equal heights;

(b) the decomposition matrix and Cartan matrix for $B$ are the same as those for the $\varepsilon^{0}$-representations of $U$;

(c) $U$ has a normal Sylow subgroup $D^{0}$ isomorphic to $D$, and $D^{0}$ contains its own centralizer;

(d) $U / D^{0} \cong S / D H$, where $S$ and $H$ were defined in section 3 .

This theorem can be interpreted as meaning that just as the part of $G$ outside $S$ can be removed without changing the structure of $B$, so the "part between $A$ and $H$ " can be removed if we allow the introduction of a factor set.

We now reformulate Theorem 5 so as to eliminate the use of projective representations, just as in the proof of Theorem 4 . Let $E$ be the character group of the cyclic group generated by $\varepsilon^{0}$. For any elements $u, v$ of $U$, let $n_{u, v} \in E$ be the character such that $\left(\varepsilon^{0}\right)^{i} \rightarrow\left(\varepsilon_{u, v}^{0}\right)^{i}$; then $n=\left\{n_{u, v}\right\}$ is a factor set of $U$ in $E$. Let $M$ be the extension of $E$ by $U$ with this factor set and trivial action; the elements of $M$ may be written $(e, u)=(e,(a, \sigma \bar{H}))$, where $e \in E . \quad E$ is in the center of $U$, and is cyclic of order prime to $p$. By defining $\mathfrak{X}_{j}^{\prime \prime}(\boldsymbol{e}, u)=e\left(\varepsilon^{0}\right) \mathfrak{X}_{j}^{0}(u)$, we set up a $1-1$ correspondence between the $\varepsilon^{0}$-representations $\mathfrak{X}_{j}^{0}$ of $U$ and a certain set $T^{\prime \prime}$ of ordinary irreducible representations $\mathfrak{X}_{j}^{\prime \prime}$ of $M$; and $\mathfrak{x}_{j}^{\prime \prime}$ is in $T^{\prime \prime}$ if and only if the linear character of $E$ contained in $\mathfrak{X}_{j}^{\prime \prime} \mid$ E can be identified with $\varepsilon^{0}$ under the canonical isomorphism between an abelian group and the character group of its character group. (For consistent notation, $T^{\prime \prime}$ should really be defined as a set of characters.) There is a similar correspondence for modular representations. Together these correspondences preserve decomposition numbers; so $T^{\prime \prime}$ is actually a block $B^{\prime \prime}$ of $M$.

To study the structure of $M$, let $\pi$ be the natural homomorphism of $M$ onto $U$. Let $H^{\prime \prime}=\pi^{-1}\left(A^{0}\right)$. Since $n \mid D$ is trivial, $\pi^{-1}\left(D^{0}\right)$ is a direct product $D^{\prime \prime} \times E=D^{\prime \prime} H^{\prime \prime}$, where $D^{\prime \prime}$ is a normal Sylow subgroup of $M . D^{\prime \prime} \cap H^{\prime \prime}$ is the center $A^{\prime \prime}$ of $D^{\prime \prime}$, and $\pi^{-1}\left(L^{0}\right)=L^{\prime \prime}$ is a $p$-complement $\pi^{-1}\left(K^{0}\right)=K^{\prime \prime}=A^{\prime \prime} L^{\prime \prime}$. Since $B^{\prime \prime}$, like every block of $M$, has the normal Sylow group $D^{\prime \prime}$ as its defect group, the theory of section 3 applies to $B^{\prime \prime}$. We need not go into detail, but simply remark that $M$ plays the role of $S$ as well as of $G$, that our notation 
for subgroups indicates their roles, and that the group corresponding to $U$ is $U$ itself (up to isomorphism). This completes the proof of the following reduction theorem for the structure of blocks with normal defect group.

Theorem 6. Let $B$ be a block of a group $G$, with normal defect group $D$. Then there exists a group $M$ and a block $B^{\prime \prime}$ of $M$ such that:

(a) the representations, both ordinary and modular, of $B$ are in $a$ 1-1 correspondence with those of $B^{\prime \prime}$, and corresponding representations have proportional degrees and equal heights;

(b) the decomposition matrix and Cartan matrix for $B$ are the same as for $B^{\prime \prime}$;

(c) $D$ is isomorphic to the defect group $D^{\prime \prime}$ of $B^{\prime \prime}, D^{\prime \prime}$ is a normal Sylow subgroup of $M$, and the centralizer of $D^{\prime \prime}$ has form $H^{\prime \prime}=A^{\prime \prime} \times E$, where $A^{\prime \prime}$ is the center of $D^{\prime \prime}$ and $E$ is a cyclic subgroup of the center of $M$;

(d) $M / D^{\prime \prime} H^{\prime \prime} \cong S / D H$.

\section{Applications of the Construction}

The following theorem treats a situation midway between that of section 2 and that of sections 3 and 4.

Theorem 7. Let $G$ be a group of form $D H$, where $D$ is the defect group of a block of $G$, and $H$ is the centralizer of $D$ in $G$. Then there is a 1-1 correspondence between the representations $\mathfrak{B}_{l}$ of $B$ and all the representations $\mathfrak{B}_{l}^{0}$ of $D$, in which corresponding representations have proportional degrees. $B$ contains just one modular representation.

Proof. $D$ is normal in $G$, so that we can use the terminology of sections 3 and 4. Here $S=D H, K=H$, and $\varepsilon$ is trivial. Therefore $U=D^{0} \cong D$, and we can identify $U$ with $D$. Theorem 5 then gives the result.

Explicitly, a short computation based on equations (15) and (17) shows that $\mathfrak{B}_{l}\left(a s_{\delta} s_{\kappa}\right)=3 m\left(s_{\kappa}\right) \times \mathfrak{B}_{l}^{0}\left(a s_{\delta}\right)$ in our usual notations. From this it is not hard to show that for $x \in D H$ we have, as a generalization of (6),

$$
\theta_{l}(x)= \begin{cases}\theta_{l}^{0}\left(x_{p}\right) \zeta(x) & \text { if } x_{p} \in D, \\ 0 & \text { if } x_{p} \notin D,\end{cases}
$$

where $\theta_{l}$ and $\theta_{l}^{0}$ are the characters of $\mathfrak{B}_{l}$ and $\mathfrak{B}_{0}^{l}$ respectively. Theorem 7 is less deep than Theorems 5 and 6 , since the proofs of these collapse considerably 
in the case $G=D H$. An alternate proof of Theorem 7 can be constructed along the lines of the proof of Theorem 3, considering $D H$ as a homomorphic image of the abstract direct product of the groups $D$ and $H$. We now return to the general case.

TheOREM 8. Let $B$ be a block of $G$ with normal defect group $D$. Then the height of each representation $\mathfrak{X}_{j}$ in $B$ is equal to $\nu\left(\operatorname{deg} \mathfrak{B}_{l}^{0}\right)$, where $\mathfrak{B}_{l}^{0}$ is any irreducible constituent of $\mathfrak{X}_{j} \mid D$.

Proof. If $\mathfrak{X}_{j}=\left(\mathfrak{X}_{j}^{\prime}\right)^{G}$, if $\mathfrak{B}_{l}$ is a constituent of $\mathfrak{X}_{j}^{\prime} \mid D H$, and if $R_{l}$ is the inertial group in $S$ of the character of $\mathfrak{B}_{l}$, then by [7] $\mathfrak{X}_{j}^{\prime}$ is induced from a representation of $R_{l}$ which is a tensor product of two projective representations, one of them being an extension of $\mathfrak{B}_{l}$, and the other being a projective representation of $R_{l} / D H$. A theorem of Schur (see [9]) tells us that the degree of the last divides $\left(R_{l}: D H\right)$, whence $\nu\left(\operatorname{deg} \mathfrak{x}_{j}^{\prime}\right)=\nu\left(\operatorname{deg} \mathfrak{B}_{l}\right)$. Since $\mathfrak{X}_{j}$ and $\mathfrak{X}_{j}^{\prime}$ have the same height, the result follows from Theorem 7 .

As an immediate corollary, we have the following result.

TheоRем 9. If $B$ has normal defect group $D$, then $B$ contains representations of positive height if and only if $D$ is non-abelian.

THEOREM 10. If the block $B$ of $G$ has normal defect group $D$, the representations of $G / D$ in $B$ are all modularly irreducible and of height 0. Taken modularly, they all remain distinct, and they yield all the irreducible modular representations of $B$.

Proof. We can reduce at once to the situation in $S$. Every irreducible modular representation $\mathfrak{F}_{k}^{\prime}$ in $B^{\prime}$ has $D$ in its kernel, and hence is a modular constituent of some $\mathfrak{X}_{j}^{\prime}$ in $B^{\prime}$ whose kernel contains $D$. Every such representation $\mathfrak{x}_{j}^{\prime}$ has height 0 by Theorem 8 ; this means that $\nu_{\nu}\left(\operatorname{deg} \mathfrak{X}_{j}^{\prime}\right)=\nu(S: D)$, so that $\mathfrak{X}_{j}^{\prime}$ is in a block of $S / D$ of defect 0 . Then all such $\mathfrak{X}_{j}^{\prime}$ are modularly irreducible, and yield distinct modular representations, as required.

The above theorems actually depend only on Theorems 1 and 7 . We conclude with a result whose proof uses Theorem 6 more fully.

Theorem 11. If $B$ has normal defect group $D$, then each Cartan invariant $c_{k l}$ of $B$ satisfies $c_{k l} \leq(D: 1)$. 
Proof. By Theorem 6, we need only prove this for the group $M$. But $M$ contains the $p$-complement $L^{\prime \prime}$, and therefore the result is given by Theorems 8 and 9 of [5].

\section{REFERENCES}

[1] K. Asano and K. Shoda, Zur Theorie der Darstellungen einer endlichen Gruppe durch Kollineationen, Compositio Mathematica vol. 2 (1935), pp. 230-240.

[2] R. Brauer, Number theoretical investigations on groups of finite order, Proceedings of the International Symposium on Algebraic Number Theory, Tokyo, 1956, pp. 55-62.

[ 3 ] R. Brauer, Zur Darstellungstheorie der Gruppen endlicher Ordnung. I, Math. Zeit. vol. 63 (1956), pp. 406-444.

[4] R. Brauer, Zur Darstellungstheorie der Gruppen endlicher Ordnung. IJ, Math. Zeit. vol. 72 (1959), pp. 25-46.

[5] R. Brauer and C. Nesbitt, On the modular characters of groups, Ann. of Math. vol. 42 (1941), pp. 556-590.

[6] R. Brauer and J. Tate, On the characters of finite groups, Ann. of Math. vol. 62 (1955), pp. 1-7.

[ 7 ] A. H. Clifford, Representations induced in an invariant subgroup, Ann. of Math. vol. 38 (1937), pp. 533-550.

[ 8 ] P. Fong, On the characters of $p$-solvable groups, Trans. Amer. Math. Soc. vol. 98 (1961), pp. 263-284.

[ 9 ] N. Ito, On the degrees of irreducible representations of a finite group, Nagoya Math. J. vol. 3 (1951), pp. 5-6.

[10] G. W. Mackey, Unitary representations of group extensions. I, Acta Mathematica vol. 99 (1958), pp. 265-311.

[11] H. Nagao, On the theory of representation of finite groups, Osaka Math. J. vol. 3 (1951), pp. 11-20.

[12] M. Osima, On the representations of groups of finite order, Math. J. of Okayama Univ. vol. 1 (1952), pp. 33-61.

[13] H. Zassenhaus, The Theory of Groups, New York, 1949.

Addendum: It is possible to carry out the constructions of sections 3 and 4 in any finite algebraic number field $\Omega$ containing the $g$-th roots of unity without enlarging this field. The proof of this depends upon the following theorem, whose proof I shall publish elsewhere.

THEOREM. Let $H$ be a normal subgroup of a group $G$ of finite order $g$, and let $\varepsilon$ be a complex-valued factor set on $G / H$. Then $\varepsilon$ is equivalent to a factor set $\varepsilon^{\prime}$ on $G / H$ whose values are g-th roots of unity, and such that for each group $G_{1}, H \subseteq G_{1} \subseteq G$, every $\left(\varepsilon^{\prime} \mid G_{1}\right)$-representation of $G_{1}$ can be written in the field of the g-th roots of unity. If furthermore $p$ is a prime which does not 
divide $(G: H)$, and if $g=p^{a} g_{r}$ with $\left(p, g_{r}\right)=1$, then we can suppose that the values of $\varepsilon^{\prime}$ are $g_{r}$-th roots of unity.

If we apply this theorem to $K$ at the point in section 3 where the $\mathfrak{Y}_{m} \mid K_{m}$ have just been constructed in an extension field of $\Omega$, we can replace $\varepsilon$ by $\varepsilon^{\prime}$ and then write $\mathfrak{Y}_{m} \mid K_{m}$ in $\Omega$. Note however that this replacement may increase the multiplicative order of $\varepsilon$, and with it the order of $M$ in section 4 . Standard methods (see in particular p. 223 of N. Jacobson, Lectures on Abstract Algebra, vol. 2, New York, 1953) then let us take $\bigvee_{m} \mid K_{m}$ locally integral in $\Omega$ and $\mathfrak{Y}_{m}^{*}\left|K_{m}=\mathfrak{Y}^{*}\right| K_{m}$. The construction of $\mathfrak{Y}_{m}$ on $S_{m}$ then proceeds in $\Omega$.

The representation $\mathfrak{Y}_{m} \times \mathfrak{H}_{j}$ in (15) is similar to a representation in $\Omega$ whose restriction to $H$ is $3_{m} \times I$; the latter representation has form $\mathfrak{Y}_{m} \times \mathfrak{R}_{j}^{\prime}$ with $\mathfrak{A}_{j}^{\prime}$ in $\Omega$, and we can replace $\mathfrak{N}_{j}$ by $\mathfrak{A}_{j}^{\prime}$ without any further change in the factor sets. A similar argument works for $\mathfrak{B}_{k}$. Then the constructions of sections 3 and 4 can be completed with all representations written in $\Omega$ and its residue class field $\Omega^{*}$.

Tufts University 